
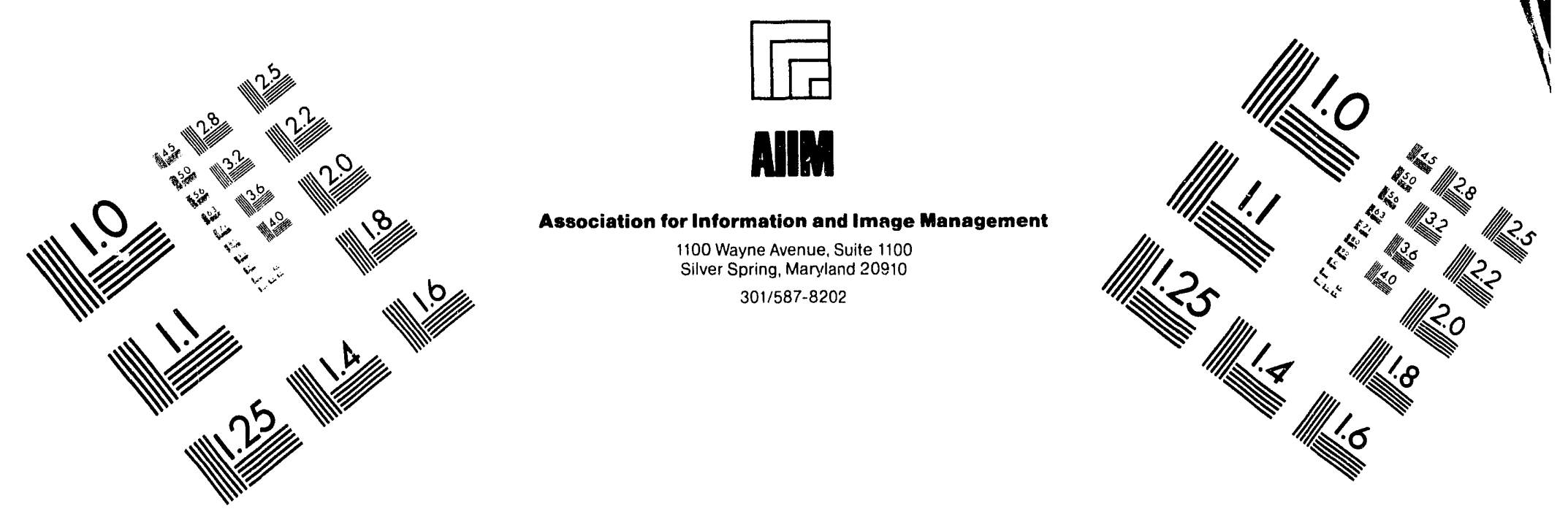

\title{
Centimeter
}

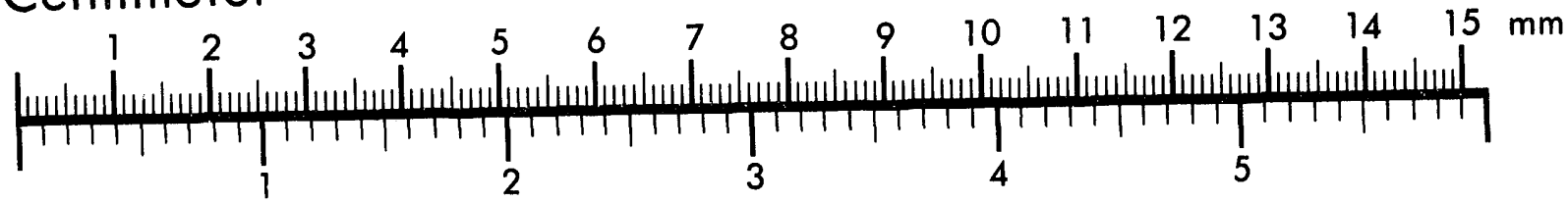
Inches
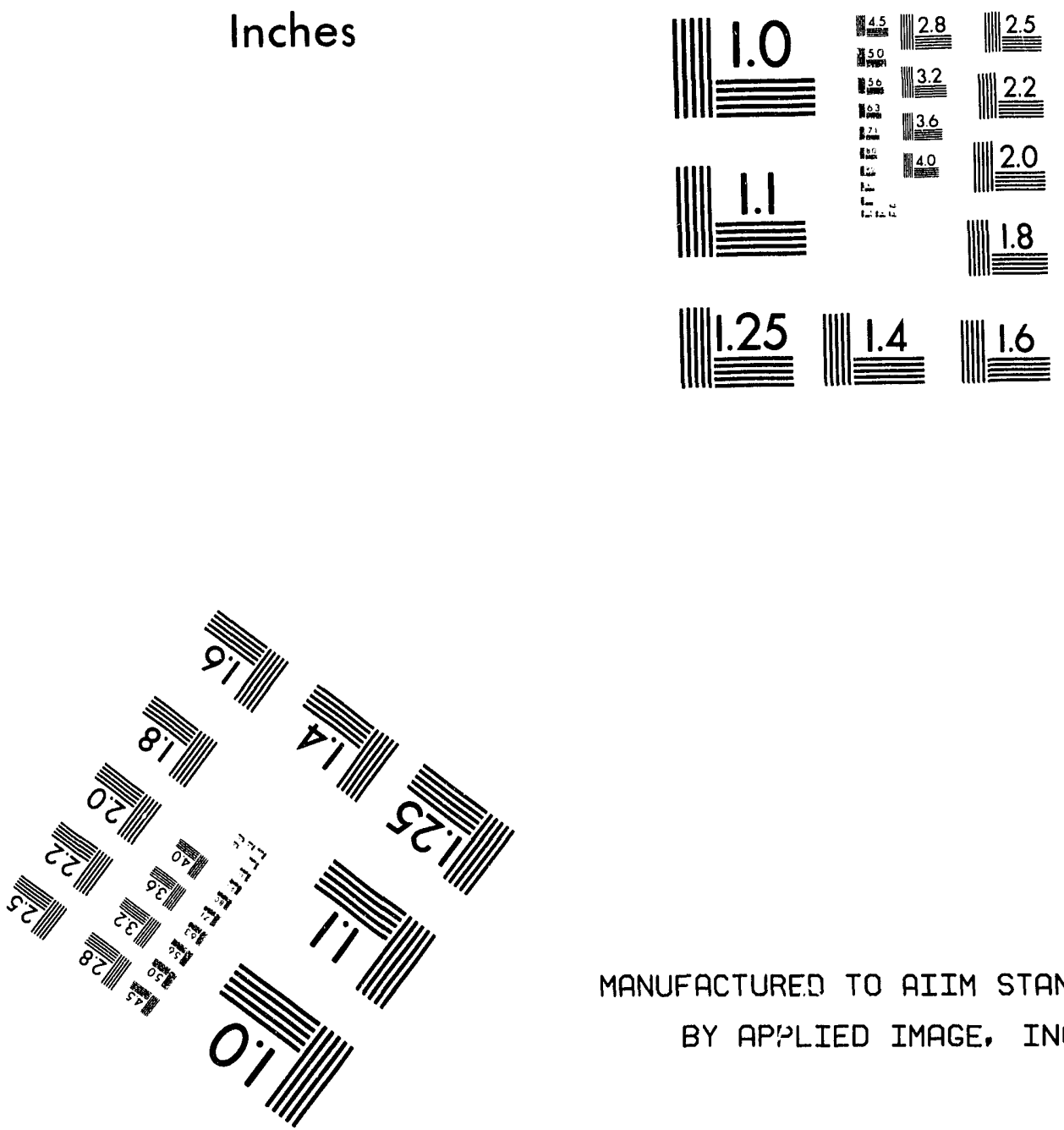

MANUFACTURED TO AIIM STANDARDS

BY APILIED IMAGE, INC.

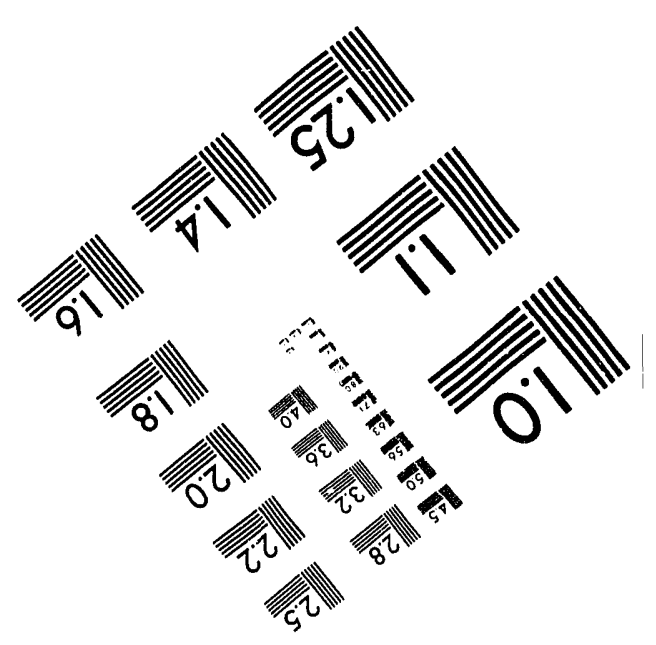



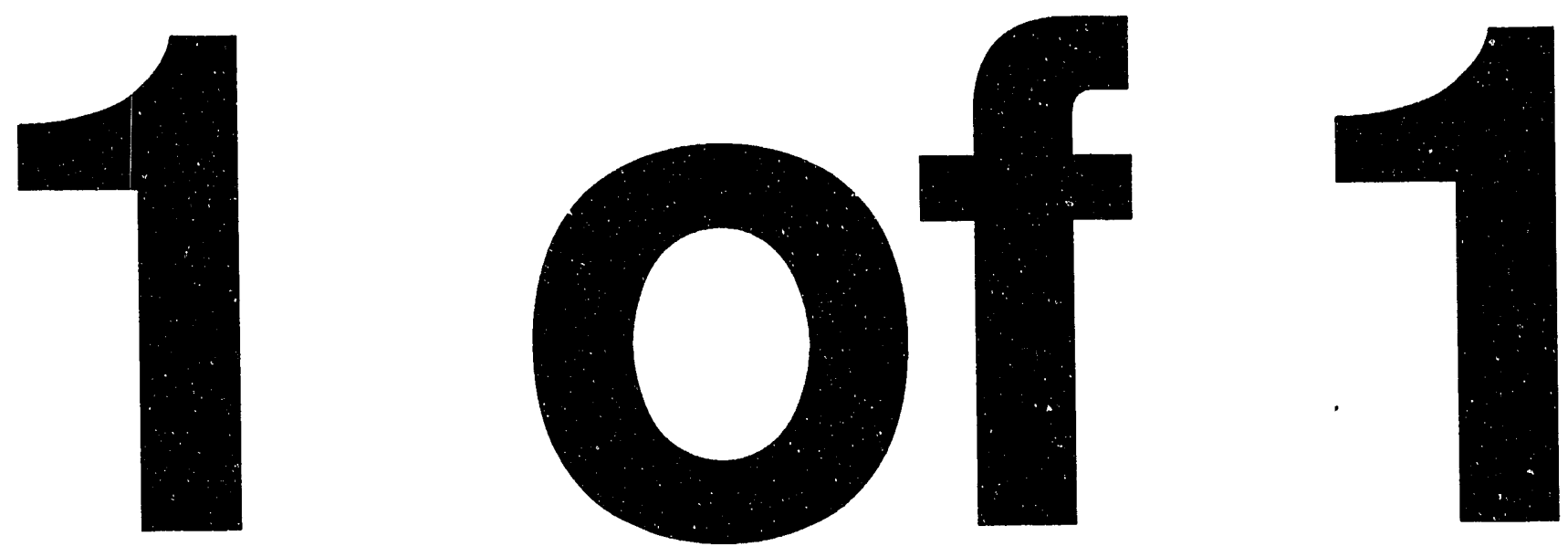


\title{
Reactions of Cresol in Hot Aqueous Borate Solutions
}

\author{
Leon Tsao and Oleh Weres* \\ Earth Sciences Division \\ Lawrence Berkeley Laboratory \\ University of California \\ Berkeley, California 94720 \\ *Now at Sonoma Research Company \\ P.O. Box 116, \\ Vineburg, CA 95487-0116
}

April 1992

\section{Project Officer}

Robert E. Smith

Underground Injection Control Board

Office of Drinking Water

U.S. Environmental Protection Agency

Washington, DC 20460

This work was supported by the Underground Injection Control Board,

Office of Drinking Water, U.S. Environmental Protection Agency, under Interagency Agreement DW89931336-01-0 between

the U.J. Environmental Protection Agency and the U.S. Department of Energy, through Contract No. DE-AC03-76SF00098. 


\section{EXECUTIVE SUMMARY}

Phenol and methylphenol (cresol) are constituents of certain waste streams being considered for underground injection. We studied reactions of these compounds in solutions with other constituents of the waste streams and suspended clay at concentrations and temperatures higher than expected in natural situations, i.e. at $200^{\circ} \mathrm{C}$ and $250^{\circ} \mathrm{C}$. Under these conditions, the predominant reaction was the demethylation of cresol to form phenol. This reaction was catalyzed strongly by clay. We were able to quantify phenol production. Other important reactions were a variety of condensation reactions in which two cresol molecules fuse. We found evidence of the intermolecular migration of methyl groups from the molecular weights of some of these condensation reactions. By digesting a sample of reacted clay with hydrofluoric acid we determined that under these conditions phenol and cresol did not bind appreciably to clay but that the condensation products did.

Our results have some practical implications regarding the underground injection of waste streams containing phenol or cresol. Most important is that phenol and cresol at the concentrations used in our experiments do not bind to clay. Since their concentrations in waste streams will likely be less we do not expect them to be retarded as the injected waste stream moves through the injection stratum. However, their condensation products do bind to clay and their migration will be retarded. We expect the reaction rates for the condensation reactions to decrease much more with decreasing cresol concentration than the rate of the demethylation reaction, because the condensation reactions are expected to be second order with respect to cresol concentration.

The rates of demethylation of cresol methyl group intermolecular migration and methanol adsorption may vary according to the mineral content of any injection formation. The suitablity of underground injection for cresol disposal will likely prove site specific. 


\section{INTRODUCTION}

The use of deep underground inj - nion as a method of disposal of toxic organic wastes must proceed from a clear understanding of the fate of these wastes once they are injected. An important aspect of their fate is the chemical reactions of the wastes in the disposal stratum. A variety of reactions may take place which render the wastes more or less toxic and more or less mobile. Constituents of the waste may react with one another, or with materials in the injection formation. Physical conditions in the formation and the chemical nature of waste stream and formation rock will affect the rate at which these reactions take place.

The compositions of waste streams and potential injection strata are too various for any single set of experimental conditions to be of general use. Each waste stream and injection stratum combination must be studied separately. Furthermore, organic constituents will usually react slowly at ambient temperatures in a deep well injection zone. Laboratory experiments at these temperatures might require years to yield measurable quantities of their products. Laboratory reaction rates can be enhanced by using higher temperatures and concentrations than occur naturally.

Our charter was to a study waste stream containing phenol and methylphenol (cresol). Cresol occurs in the waste water from certain steel processes in which borate esters of cresol are used as lubricants. Borate is another significant component of the waste stream we were asked to study. The waste stream containing phenol comes from a process that was not identified to us, but contains ammonium sulfate as a significant component. The injection zone rock was not specified for this study.

Phenol is a derivative of benzene having a hydroxyl group substituted for a hydrogen atom. This alters the reactivity of the benzene ring substantially, making substition reactions easier and condensation reactions possible. In the context of this study, condensation, the binding of one phenol molecule to another, is an important type of reaction. Phenol behaves as a weak acid and forms alkali metal salts. Cresol is the common name for the mixture of ortho-, meta-, and paramethyl derivatives of phenol, which is available commercially. The methyl phenols are similar to phenol in their acidic and alcoholic properties but the presence of the methyl group alters their reactivities. Cresol also behaves as a weak acid. Because the methyl group donates a negative charge to the molecule as a whole, the anion is less stable relative to the uncharged species when compared with the phenol anion. Therefore, cresol is a slightly weaker acid with higher $\mathrm{pK}_{\mathrm{a}}$ than phenol. It also behaves as an alcohol and forms esters with acids. The important ester, for the purview of this paper, is cresyl borate. 
The tricresyl ester of boric acid is used industrially and the presence of its decomposition products in some industrial water is the ultimate motivation for this research. Although this ester is not stable in water it is possible that a monocresyl ester of boric acid has a fleeting existence that allows it to serve as a reaction intermediate. Boric acid is highly electrophilic with a strong propensity toward completing its electronic octet (Gerrard,1961). The cresol molecule with its methyl group provides a source of electrons. As an electrophile it would be more likely to attach to the cresylate anion form than the un-ionized form.

Most studies involving cresol have been done under conditions relevant to industrial processes, and not under those relevant to waste product disposal. Typically, experiments reported in the literature involved 33 to 100 mole percent phenol in the gas phase above $400^{\circ} \mathrm{C}$.

The demethylation of cresol has been studied at temperatures between $390^{\circ}$ and $440^{\circ} \mathrm{C}$ with an initial system of pure cresol by Delaunois and Godefroid (1970). Under these conditions they observed dehydroxylation as well as demethylation of cresol. They also reported the formation of xylenes and xylenols, indicating that the transfer of methyl groups took place. Smaller quantities of condensation products such as diphenyl, dibenzyl and dibenzofurane were also produced. Even under reaction conditions substantially different from our own both condensation and methyl group migration occur.

\section{REACTIONS OF CRESOL AND PHENOL}

\subsection{Experimental Conditions}

We ran our experiments at $200^{\circ}$ and $250^{\circ} \mathrm{C}$ to increase reaction rates and allow reasonable reaction times. Because we expected it to be the most reactive cresol isomer we used $+99 \%$ paramethyl phenol. Using a single isomer also simplifies the task of identifying reaction products. Most experiments ran for 72 hours in $600 \mathrm{ml}$ titanium autoclaves, manufactured by Parr Instruments, and equipped with magnetically coupled stirrers, which eliminated possible leakage.

In the earliest series of experiments with cresol a typical reaction mixture consisted of $18.00 \mathrm{~g}$ of reagent grade $\mathrm{Na}_{2} \mathrm{~B}_{4} \mathrm{O}_{7} \cdot 10 \mathrm{H}_{2} \mathrm{O}, 5.70 \mathrm{~g}$ of reagent grade $\mathrm{NaCl}$, $5.00 \mathrm{~g} \mathrm{p}$-cresol (Aldrich Gold label), $50.0 \mathrm{~g}$ bentonite clay from commercial drilling mud pellets, and $341 \mathrm{ml}$ distilled $\mathrm{H}_{2} \mathrm{O}$. The drilling mud was checked for organic content using a Perkin-Elmer $240 \mathrm{C}$ elemental analyzer. It contained $0.25 \%$ carbon by weight which could be either organic or carbonates. Solvent extraction of a blank run 
showed no detectable organic compounds extractable into methylene chloride. In later experiments, when it was our intention to elicit kinetic information, we reduced the clay in the charge to $35.0 \mathrm{~g}$ to produce a thinner mixture which was easier to handle.

After closing the vessel we flushed the head space with helium and pressurized it to approximately $16 \mathrm{psig}$. This was done to provide a non-reactive atmosphere and a carrier gas for collecting gaseous reaction products.

At the end of 72 hours we allowed the reaction vessel to cool and collected the head space gases over water in a separatory funnel. These were then analyzed on a Consolidated Engineering Corp. 21-102 mass spectrometer. The vessel was then opened and the contents decanted into plastic jars for centrifugation. After centrifugation to separate the clay, we removed the supernatant solution and put $50 \mathrm{ml}$ in a separatory funnel along with $10 \mathrm{ml}$ of gas chromatography grade methylene chloride $\left(\mathrm{CH}_{2} \mathrm{Cl}_{2}\right)$. After vigorous agitation the aqueous solution was removed and the methylene chloride washed once with distilled water and filtered through anhydrous sodium sulfate to remove traces of water. The methylene chloride extract was stored in an EPA approved vial with a Teflon lined cap, previously rinsed with methylene chloride.

We analyzed extracts of reaction products on a Hewlett-Packard 5880 gas chromatograph (GC) and later on a Perkin-Elmer $8500 \mathrm{GC}$, to obtain reliable peak areas to develop estimates of the mole fraction of starting material consumed in various reactions. We also used a Finnigan $4000 \mathrm{GC} / \mathrm{mass}$ spectrometer. With the latter instrument we could identify organic compounds. Where perdeutero analogs were available we could quantify the yield of certain compounds by spiking the reaction product solution with an appropriate perdeutero compound and comparing the areas of mass chromatograms.

\subsection{Results}

After performing a blank experiment with the vessel and inorganic materials, we ran an experiment with the typical earlier charge, with $50 \mathrm{~g}$. of clay to determine which reactions took place. We discovered in our first experiment that the single most important reaction was the demethylation of cresol to form phenol. After subsequent experiments we were able to quantify phenol production by adding a spike of perdeutero phenol. Phenol accounted for $1.4 \%$ of the initial cresol.

There were also a number of condensation products. We estimate these to account for $2.1 \%$ of initial cresol, based on the areas of chromatographic peaks. The $\mathrm{pH}$ dropped from an initial value of 9.12 to 6.15 . Analysis of the product gases 
indicated that small quantities of hydrogen, methane and carbon dioxide were produced. The significance of variations in these quantities depending on reaction conditions will be discussed later. The methane produced could account for only an insignificant portion of the methyl groups taken off the cresol molecules. It seemed important to know if methanol, itself toxic, was an important reaction product.

To quantify the methanol yield we performed a series of spiking and extraction experiments using perdeuteromethanol, and developed a series of standards for comparing methanol and perdeutsromethanol concentrations. We distilled $25 \mathrm{ml}$ aliquots of product solution, taking the first three drops to come over after an hour of refluxing. Analysis on the GC/MS by comparison of the intensities of characteristic mass fragments showed that methanol accounted for between $1.4 \%$ and $4.4 \%$ of all methyl groups removed from cresol. We discuss below another process which accounts for more but not all methyl groups removed from cresol.

When we ran the experiment without clay we discovered that clay very strongly catalyzed the demethylation of cresol and weakly catalyzed its condensation reactions. When we ran the experiment without borate we discovered that most of the condensation reactions were catalyzed in its presence. Here we must be careful to state that our work to date does not allow us to distinguish what may be catalysis by borate ions from catalysis by hydroxide ions whose concentration will be strongly influenced by a buffer of boric acid salts. Gas chromatograms of the product extracts from the three runs are shown in Figure 1.

The amounts of unreacted cresol, phenol and condensation products produced by these two experiments, expressed as a fraction of the mass of initial cresol are given in the table below.

Table 1. - Reaction products

as percentage of initial cresol

\begin{tabular}{|l|c|c|}
\hline & no clay present & clay present \\
\hline unreacted cresol & 97.3 & 90.7 \\
\hline phenol & 1.1 & 3.7 \\
\hline condensation products & 1.6 & 5.6 \\
\hline
\end{tabular}


$-7$.

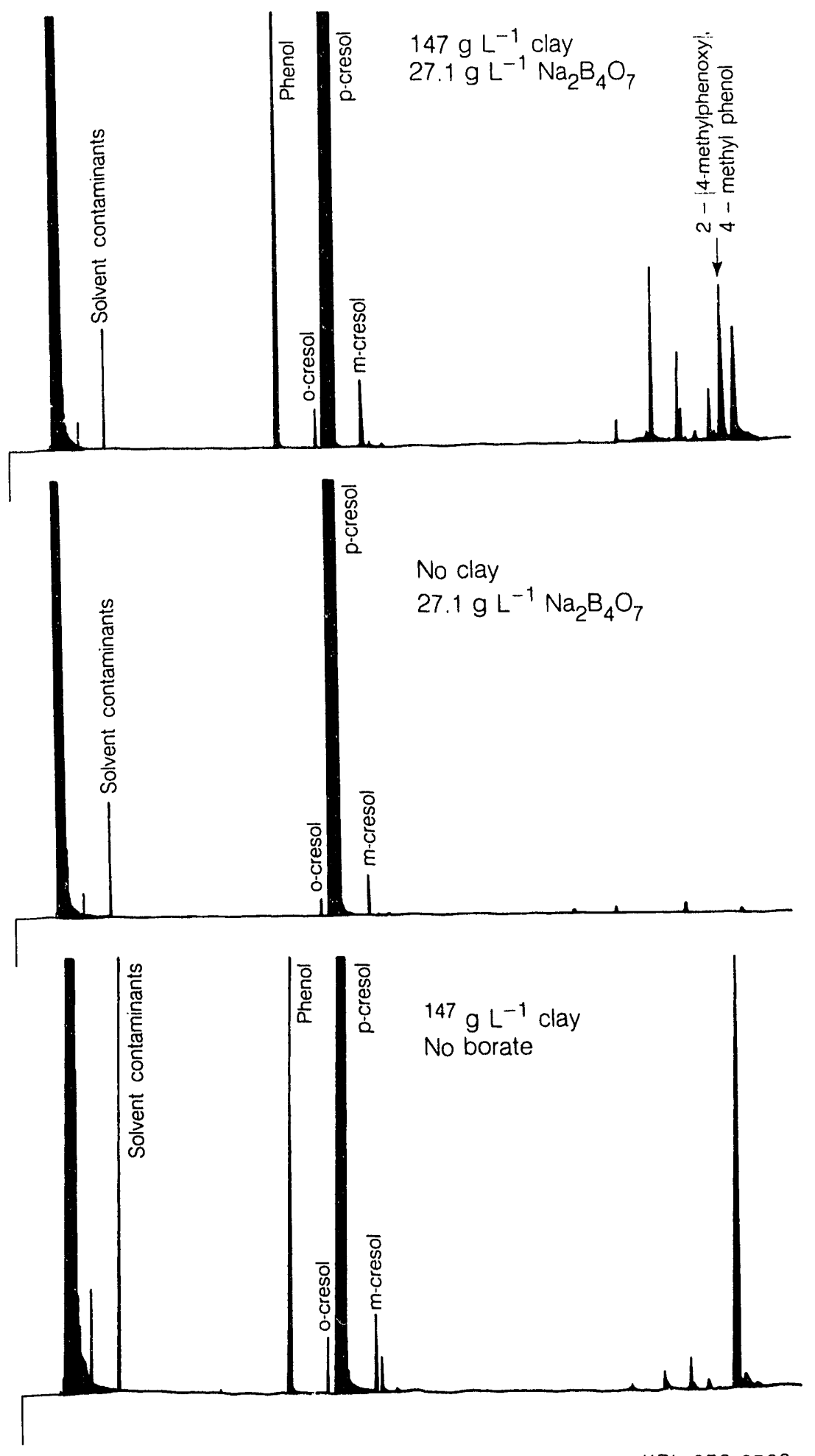

XBL $878-3722$

Fig. 1 Chromatograms of product solutions from different reaction conditions. 
The gas products of the experiment without clay contained no measurable carbon dioxide. This suggests that carbon dioxide produced in other experiments comes from trace carbonates in the bentonite. The experiment without clay produced roughly 50 times as much hydrogen as either the experiment with clay and borate or with clay only. This may be due to hydrolysis. This observation suggests that hydrogen is produced by corrosion of the autoclave or by reactions catalyzed by the metal surface and that either clay inhibits this reaction or consumes hydrogen through a redox reaction in which ferric ions are reduced to ferrous ions. It is possible that some of the many organic reactions occuring in this system consume hydrogen.

In order to understand the kinetics of the reactions of cresol, we decreased the amount used to $31.6 \%$ of that used in the initial experiments. The amount of phenol produced was reduced to $58.2 \%$ of that produced in the initial experiments. This observation is consistent with $1 / 2$ order kinetics, because $(.582)^{2}=0.339 \cong 0.316 .1 / 2$ order kinetics would be expected of a free radical chain reaction. Considering that boric acid or hydoxide ions may also influenced the reaction, and that the $\mathrm{pH}$ changed over the course of the reaction, the experimental conditions were not controlled well enough to draw any clear inference.

Using GC/mass spectrometry we were able to identify only two of the condensation products with confidence. One was 2-[4-methylphenoxy],4-methyl phenol with a molecular weight of 214.27. This was also the only condensation product whose formation was not catalyzed by boric acid or hydroxide ions. The other species we identified was 2,2' methylenebis(4-methylphenol). We found it among the products of a reaction run at $200^{\circ} \mathrm{C}$ but not among the products of the reaction at $250^{\circ} \mathrm{C}$. This serves to remind us that extrapolation of reactions to lower temperatures must be done with care. The mass spectra and structures of these compounds are given in Figure 2.

The molecular weight of cresol is 108.14 so that the simplest condensation products of two cresol molecules will have a molecular weight of 214.27. Two hydrogen atoms are displaced, accounting for the difference in mass between two cresol molecules and one of the condensation products. For some of the condensation products the molecular weight was 200 suggesting either that a cresol and a phenol molecule had fused or that a condensation product molecule was subsequently demethylated. Other products had molecular weights of 228, 242, 256 and 270, suggesting that methyl groups had migrated from a cresol molecules not involved in the condensation reaction. 

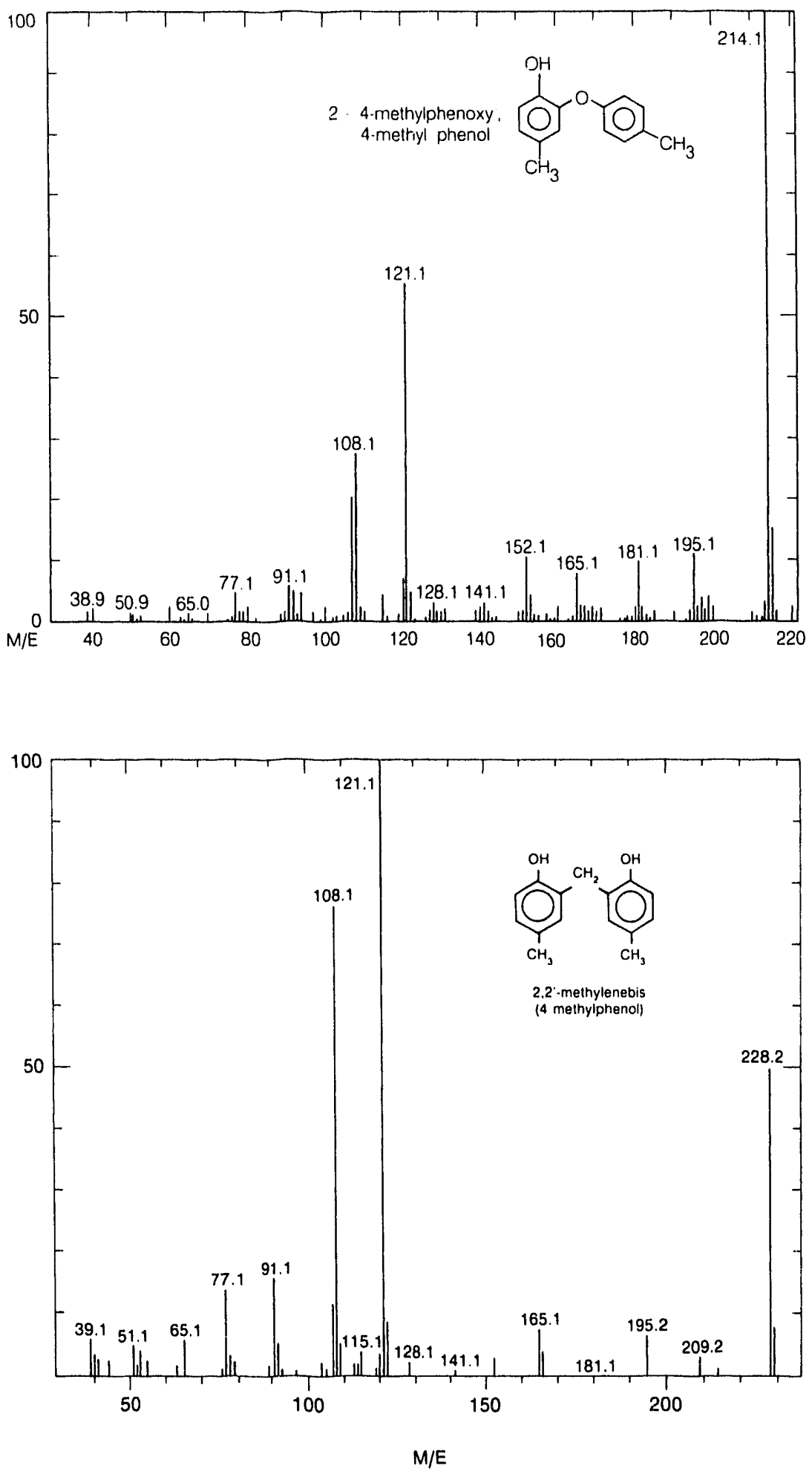

XBL 881-9519A

Fig. 2 Mass spectra and structures of two condensation products. 
When we attempted to determine what percentage of methyl groups had migrated to condensation species we checked the starting material for polymethylphenols. Unfortunately it turned out to contain a significant amount, on the order of $3 \%$, of a dimethylphenol. Therefore, any species of molecular weight 242 may be the result of condensation of dimethylphenols as well as methyl group transfer. However, the species of molecular weight 270 is clear evidence of methyl group migration.

We ran one experiment with clay that had been acid washed. The process also removed unquantified portions of the lightest and heaviest clay particles. When we analyzed the products with the GC/MS we discovered that most of the cresol had been converted to 1-methoxy-4-methylbenzene. A small amount of methoxybenzene was also produced and a small amount of cresol remained. Small amounts of unidentifiable condensation products were also produced, many with molecular weights of 242 .

We performed an experiment using phenol instead of cresol and ammonium sulfate instead of sodium borate. The product solution was worked up in the same way as the cresol experiments. Analysis by gas chromatograph revealed that the yield of all condensation products was much smaller than for reactions of cresol. The amounts of condensation products from these conditions were near the limit of detection for the HP 5880.

In order to determine the extent to which the various organic chemicals in the product solution bound to clay, we performed decompositions of washed clay, using hydrofluoric acid. Reacted clay recovered after centrifugation was resuspended in distilled water to remove loosely associated solutions and centrifuged again. This process was repeated twice. The washed clay was air dried at room temperature. We mixed 1.0 $\mathrm{g}$ of the clay with $2.0 \mathrm{ml}$ a $50 \%$ solution of trichloro-acetic acid and $10 \mathrm{ml}$ of $50 \%$ HF. This mixture was warmed gently on a hot plate and allowed to stand for four hours in the fume hood to decompose the clay to silicon hexafluoride and allow this volatile product to evaporate. The remaining residue was resuspended in water and the suspension extracted with methylene chloride and the extract analyzed by gas chromatography. Comparison of peak areas of the extract from the decomposed clay with extract from un'reated clay revealed that phenol did not bind to the clay, that cresol bound only slighly but that the condensation products did bind appreciably. Chromatograms of the two extracts are shown in Figure 3. 


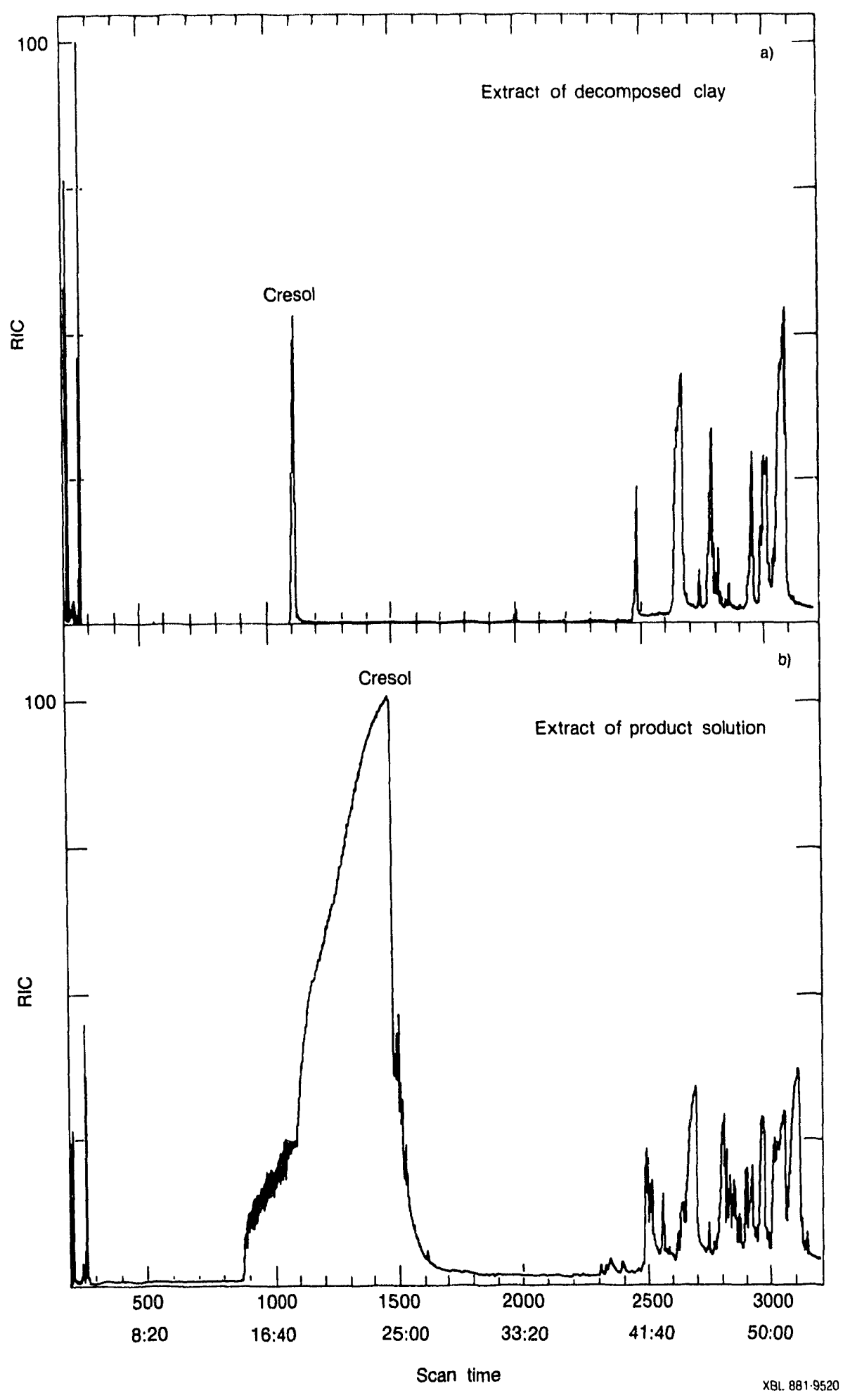

Fig. 3 Chromatograms of extracts from product solution and from decomposed clay. 


\section{DISCUSSION AND SUMMARY}

Our results have some practical implications regarding the deep underground injection of waste streams containing phenol or cresol. Most important is that phenol and cresol at the concentrations used in our experiments do not bind to clay. Since their concentrations in waste streams will likely be less we do not expect them to be retarded as the injected waste stream moves through the injection stratum. However, their condensation products do bind to clay and their migration will be retarded. It is necessary to study the low temperature kinetics of this reaction to learn how much this reaction may be relied on in planning for safer injection. Our work suggests that the demethylation reaction is $1 / 2$ order with respect to cresol concentration and the stoichiometry of the condensation reaction suggests that it is second order in cresol. We expect that the reaction rate for condensation reactions to decrease much more with decreasing cresol concentration than the rate of the demethylation reaction because the condensation reactions are expected to be second order with respect to cresol concentration.

The isomerization or intramolecular migration of methyl groups in cresols has been studied by Baddeley (1943) and by Meisner and French (1952). Baddeley also discussed intermolecular migration of methyl groups. According to Baddeley, the mechanism of migration involves first the formation of cresylaluminum chloride, which is an analog of cresyl borate from cresol and aluminum chloride, a strong electrophile. Two additional molecules of aluminum chloride become attached to the cresol group, one to the ring carbon with the methyl group bound to it, and another to the adjacent ring carbon. The methyl group then migrates from one ring carbon to the adjacent one. If this mechanism is correct for cresol in boric acid solutions we expect the rate of methyl group migration to be more strongly dependent on boric acid concentration than the rate of condensation reactions. Metacresol undergoes the least amount of migration, due to the electronic structure of the metasresyl ion. A resonance structure which allows for a tetrahedral configuration of the ring carbon to which the methyl group is bonded is only possible with the para- and ortho- isomers of ring substituted phenols. The tetrahedral configuration is necesary for methyl group migration and demethylation, if that reaction proceecis as an electrophilic substitution of hydrogen for methyl.

The aluminum salts of various alkyl substituted phenols catalyze alkyl group transfers between these molecules. When considering the catalyic role of bentonite clay in the demethylation of cresol it should be recalled that the principal constituent of bentonite is montmorillonite. Montmorillonites are aluminum-magnesium silicates of 
varying aluminum content. They also admit polar or ionic organic compounds between their layers. (van Olphen,1977) The possible reaction paths are complex since cresol probably forms silicic acid esters as well as bonds to alunininum. The geometry of the binding sites will also be important. The demethylation of cresol demonstrated in our experiments raises the question of the creation of methanol, another toxic organic compound. A more complete accounting of the methyl groups removed from cresol needs to be made. Determining whether a substantial portion of these groups ends up as methanol bound to clay is a question for future investigation. This question wilı also be important for modeling the retardation of methanol in the injection zone. The rates of demethylation of cresol and methanol adsorption may vary according to the mineral content of any injection formation. The suitablity of underground injection for cresol disposal will likely prove site specific.

\section{Acknowledgments}

We wish to thank Amos Newton for analysis of gas samples, assistance in operating the gas chromatograph/mass spectrometer and advice on developing analytical methods.

This work was supported by Interagency Agreement DW89931336-01-0 between the U.S. Environmental Protection Agency and the U.S. Department of Energy under contract AC03-967SF00098. 


\section{Bibliography}

Baddeley, G., 1943. The Action of Aluminum Chloride on Some Phenol Homologues. J. Chem. Soc., pp. 527-31

Delaunois, C., and Godefroid, J.R., 1970. Kinetic study of cracking of pure cresols under pressure in liquid phase. Ann. Mines Belg., 12, pp. 1409-22

Gernard, W., 1961. The Organic Chemistry of Boron. Academic Press, New York, p. 11

Meissner, H.P., and French, F.E., 1952. Isomerization of the Cresols by Aluminum Chloride. J. Am. Chem. Soc., 74, pp. 1000-003.

van Ophen, H., 1977 An Introduction to Clay Colloid Chemistry Wiley Interscience, New York, p. 67 

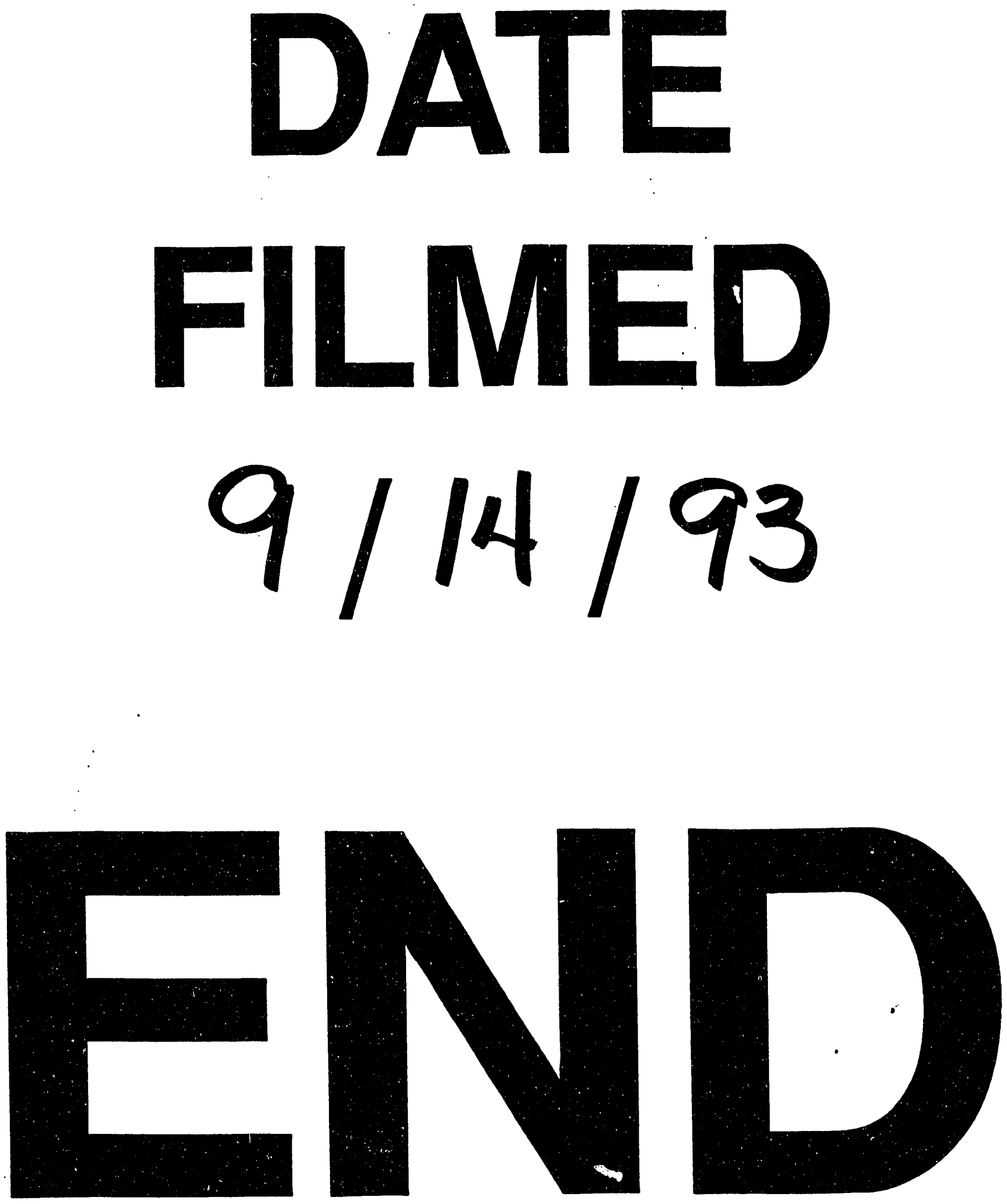
